\title{
Autoradiographical Study of Postnatal Neurogenesis in the Periventricular Regions of the Avian Brain
}

\author{
T. P. Pessacq Asenjo, L. A. Vidal Rioja and N. O. Bianchi
}

Museo de Ciencias Naturales, Univ. Nac. de La Plata, La Plata, Argentina

Received September 12, 1974

The study of post natal neurogenesis by means of histoautoradiographical procedures has received considerable attention (Uzman 1960, Fujita 1964, 1967, Hayashi 1966, Altman and Das 1966, Pessacq et al. 1972, Pessacq et al. 1968). However, a survey of the bibliography reveals the scarcity of data on many post natal histogenetical aspects of the brain development. In avian brains it is constantly observed the existence of closely applied neurons constituting a sort of nests of three or four elements. Although we can speculate that these associations could be originated by repeated post migrational divisions of a single neuroblast this fact cannot be clarified until the employment of histoautoradiographical procedures. The main purpose of the present work was to study in autoradiograms, the histogenesis of these neuronal groups in periventricular regions of the chick neostriatum, where they are particularly numerous. Although our attention was focused in this problem, some aspects of glia cells histogenesis were also analyzed.

\section{Material and methods}

5-day old chicks were injected intracranially with $20 \mu \mathrm{c}$ of tritiated thymidine (HTdR) (Sp. Act. $6.9 \mathrm{C} / \mathrm{mM}$ ) dissolved in $0.1 \mathrm{ml}$ of physiological salt solution. Chicks were inoculated through the anterior fontanelle using a $1 / 2$ in. long needle. Post mortem examination of animals showed that in every cases the injection had fallen in the cerebellar cisterne. At 1/4, 1, 4, 16 and 24 hour intervals and at 2, 3, 4 and 7 days, 3 to 4 animals inoculated at 5 days of age were sacrificed by decapitation. The brain was dissected out, fixed in 3:1 ethanol: acetic, and histologically processed. Slides were mounted with AR-10 Kodak stripping film, exposed for 7 days, developed and stained through the film with Giemsa stain.

\section{Results}

The present histoautoradiographical observations are grouped as follows: 1) those on labelled elements of the ependymal layer; 2) those on labelled periventricular neuronal nests and 3 ) those on labelled satellite cells of well differentiated isolated neurons.

1) The ependymal periventricular layer of the newly hatched chick is considerably thick and retains the same histological appearance of the matrix lamina of the embryo. It consists of large part of tightly packed roundish nuclei approximately $5 \mu$ in diameter. At the ventricular surface there is a thin slightly refractile 
line constituted by the face borders of the ependymal cells. The accumulation of radioactive cells in the periventricular region is shown in Fig. 1. This autoradiogram was obtained from a chick sacrificed 1 hour after the injection. It may be seen that most labelled cells are concentrated in the ependymal layer. A fewer number of radioactive cells were seen in the subependymal layer. In animals with longer survivals (4 days) the larger part of labelled cells is all ways situated in the ependymal layer although a considerable silver grain dilution is to be observed. It is interesting to note that the ependymal labelled cells are arranged in nests or groups, constituting perhaps synchronized cellular populations (Fujita 1964).

2) The neuronal nests of the cerebral hemispheres of Avis consist of two to

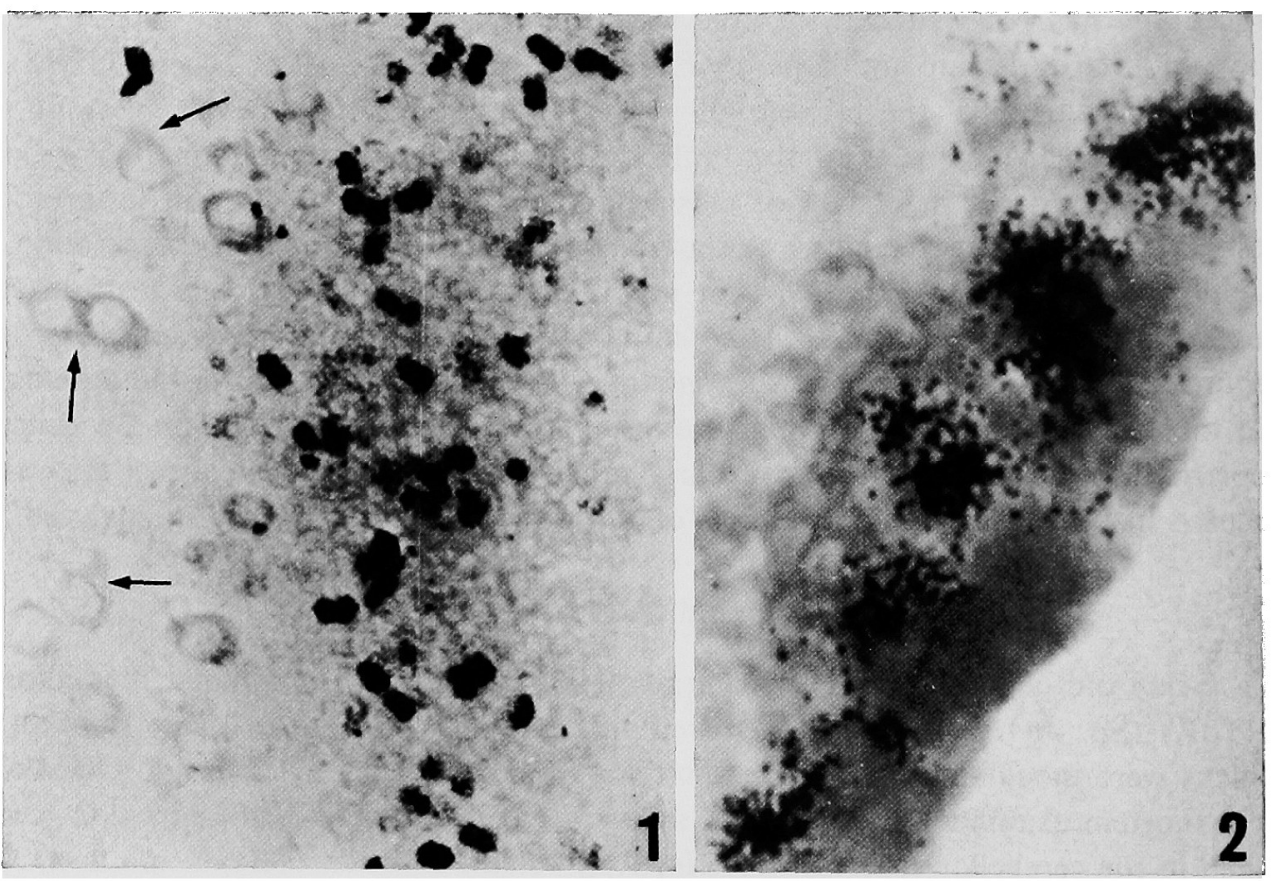

Figs. 1-2. 1, ependymal layer of the brain of a chick sacrificed 1 hour after the injection of tritiated thymidine, showing numerous labelled elements. The arrows point out subependymal isogenic groups. $\times 150.2$, labelled elements of the ependymal layer of the brain of a chick sacrificed four days after the injection of tritiated thymidine.

five neurons clossely applied. We have celled them isogenic groups (Pessacq et al. 1968). Commonly the associated adjacent neurons are uniform in size but in some cases there is a great cell accompanied by other smaller neurons. We have found three main labelling patterns:

a) Displaced neuroblastic nests constituted by several highly labelled elements.

b) Isogenic groups with a differentiated element showing in some cases a residual labelling, surrounded by highly labelled smaller cells. They offer a very characteristical shape as illustrated in Fig. 3a.

c) Isogenic groups constituted by differentiated great neurons, having among them a small labelled element. By reason of the scarcity of ctyoplasmic details it 
is in most cases difficult to speculate about the cellular specificity (glial or neuronal) of these labelled cells, although in some cases it is clear that they are neurons of
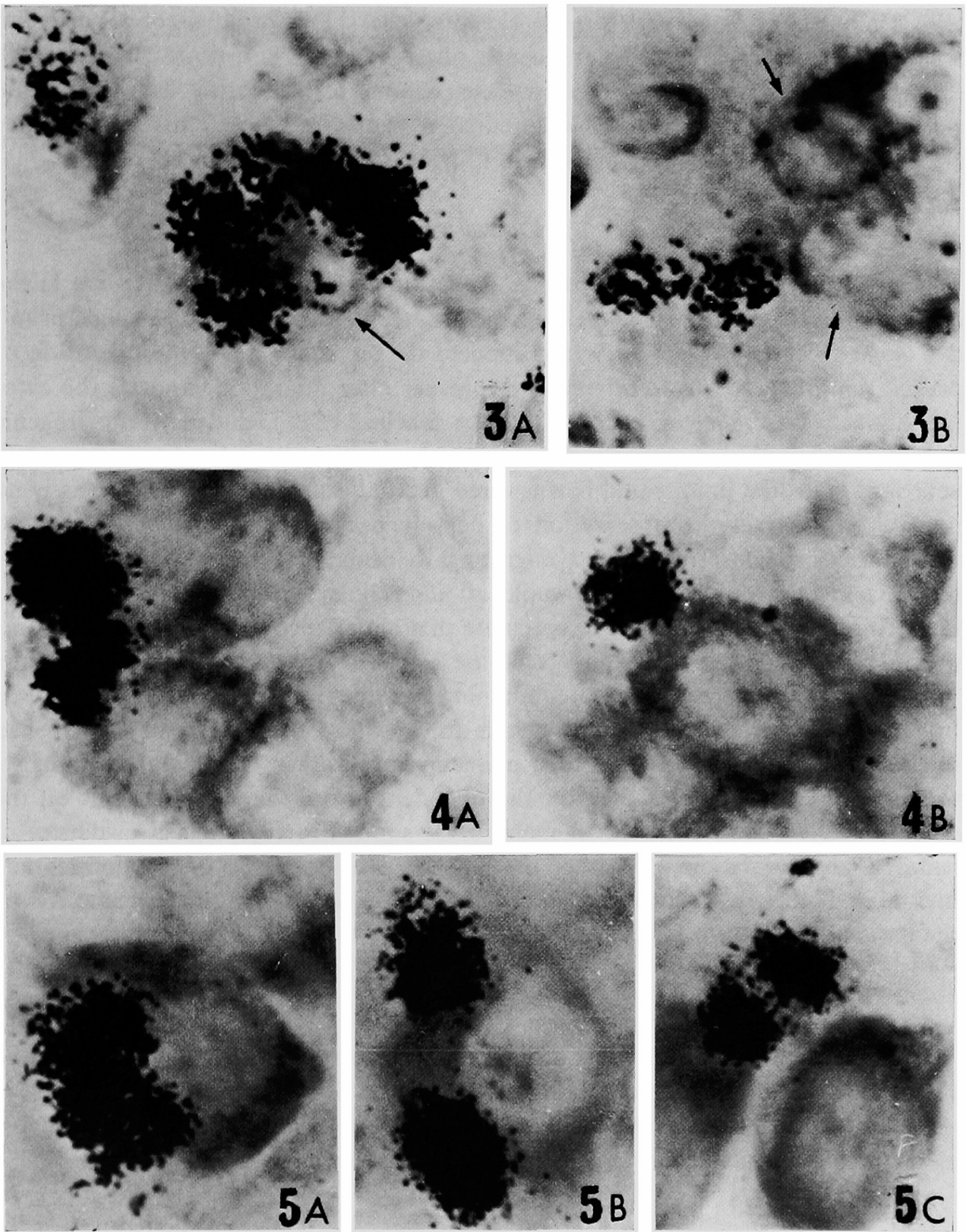

Figs. 3-5. 3 A, a group of intensely labelled elements surrounding a neuron (arrow) placed immediately below the ependymal layer. Animal sacrificed two days after the injection of tritiated thymidine. B, two labelled nuclei with grain dilution in the vicinity of a isogenic group (arrows). Animal sacrificed three days after the injection. $\times 750.4 \mathrm{~A}$ and $\mathrm{B}$, labelled elements within fully differentiated isogenic groups. Those appearing in A suggests cellular division. Animal sacrificed three days after the injection of tritiated thymidine. $\quad \times 1000.5 \mathrm{~A}, \mathrm{~B}$ and $\mathrm{C}$, labelled double neuronal satellites. $\mathrm{A}$ and $\mathrm{C}$ illustrate cellular division pictures. Animal sacrificed two days after the injection. $\times 1000$. 
small size in way of differentiation. The finding of a pair of labelled elements with apparent grain dilution in animals sacrificed four days after the injection, indicates that cellular division has taken place in the intimity of the isogenic group.

3) Single neurons with double satellite cells. This group is of a particular type of cellular association very frequently observed and is constituted by two small elements with a densely labelled nucleus having scarse perinuclear cytoplasm, closely applied to an adult well differentiated neuron. Nuclear division pictures are frequent in animals surviving two to four days after the injection (Fig. 5).

\section{Discussion}

Two main aspects of the observed facts must be discussed. They are I) the interpretation of the origin of the isogenic groups by the division of a single primitive element which divides after migration to deeper zones or by the migration of packed neuroblasts, which differentiate together after migration; and II) the cytological specificity (glial or neuronal) of the labelled cells included within isogenic groups of differentiated neurons, and the double satellite cells related to independent neurons. The first point could be answered in an affirmative fashion for the second possibility. In fact the observation of densely packed labelled nuclei, displaced from the ventricular surface and migrating to deeper regions is quite frequently recognized. Fig. 3 is a good example of this cellular arrangement. Concerning the second point it is tempting to speculate that simultaneously with the migrating process, only some of the members of the isogenic group begins to differentiate. The others remain in a blastic state, retaining thus the possibility of DNA synthesis. In most cases we lack cytological details for a solid classification of the labelled satellite elements. By reason of their perineuronal position, many neurohistologists will be tempted to include them in the oligodendrocytic population. But on the other hand, we have depicted in many cases some clear cytological evidences of neuronal differentiation. Whether the labelled satellite elements can differentiate in a double sense (perineuronal glial cells or microneurons) can not be answered on the basis of the present findings.

\section{Summary}

The histogenesis of the nests of intimately associated neurons (isogenic groups) of the avian brain is autoradiographically studied. The cellular specificity (glial or neuronal) of the labelled cells included within differentiated isogenic groups as well as the labelled double satellite cells of individual neurons is briefly analized.

\section{References}

Altman, J. and Das, G. D. 1966. Autoradiographic and histological studies of postnatal neurogenesis. I. A longitudinal investigation of the kinetics, migration and transformation of cells incorporating tritiated thymidine in neonate rats, with special references to postnatal neurogenesis in some brain regions. J. Comp. Neurol. 126: 337-390.

Fujita, S. 1964. Analysis of neurone differentiation in the central nervous system by tritiated thymidine autoradiography. J. Comp. Neurol. 122: 311-328. 
- 1967. Quantitative analysis of cell proliferation and differentaition in the cortex of the postnatal mouse cerebellum. J. Cell Biol. 32: 277-288.

Hayashi, T. 1966. Autoradiographic studies on the histogenesis of the chick cerebellum. J. Kyoto Pref. Univ. Med. 75: 1225-1239.

Pessacq, T. P. and Reissenweber, N. J. 1968. Unpublished observations. Instituto de Investigacion de Ciencias Biologicas (Montevideo).

-, Vidal-Rioja, L. and Bianchi, N. O. 1972. Autoradiographic study of the postnatal cerebellar neurogenesis in the chick. Cytologia 37: 575-580.

Uzman, L. 1960 . The histogenesis of the mouse cerebellum studied by tritiated thymidine uptake. J. Comp. Neurol. 114: 134-159. 